\title{
Avant-propos des éditrices et éditeurs de la série
}

Cette série de livres intitulée Appearances: Studies in Visual Research est née d'une collaboration de longue date entre ses éditeurs. En plus de plusieurs publications dans le domaine, ils ont organisé avec succès un grand nombre de panels et d'activités très appréciés à plusieurs conférences ISCHE (International Standing Conference for the History of Education), des évènements qui ont porté sur un large éventail de thèmes et de questions méthodologiques autour de la photographie et du cinéma en tant que sources historiques et objets d'archives.

De nos jours, les images sont partout, et chacun peut s'essayer au métier de photographe documentaire. Mais comment donnons-nous un sens à cette révolution visuelle dans la longue histoire de l'utilisation du matériel visuel comme outil de communication? Récemment, les historiens et les spécialistes en sciences sociales ont commencé à travailler sur l'intersection de la recherche visuelle, de l'histoire des médias et des études matérielles. Leurs recherches se sont particulièrement concentrées sur le fait que les images elles-mêmes ont une histoire ou une biographie sociale. Les images sont reproduites, diffusées et consommées d'une manière qui ne pouvait être prédite au moment de leur production originelle.

Les images sont incluses et inscrites dans différents contextes ; elles passent des caméras, des toiles et des papiers aux albums, plaques, musées, cadres, boîtes, murs, cartes, livres, revues, magazines populaires, catalogues, brochures, et à presque toutes les surfaces imaginables, y compris les écrans qui sont déployés par les médias numériques et les réseaux sociaux. Les technologies visuelles en tant que pratiques matérielles impliquent une impulsion pour la reproduction et la diffusion où les images assument un rôle herméneutique. Les images sont des objets reproductibles et mobiles qui ne cessent d'atteindre le public et de rassembler une grande variété de relations et de significations entrelacées au fil du temps. Les qualités matérielles et sociales des images sont donc indissociables ; les deux se réfèrent à des processus de création de sens en chaînes de reproduction, de restauration et de re-contextualisation, les images assumant des rôles actifs en tant que connecteurs et communicateurs.

Les images soulèvent des questions sur les relations entre les médias, la vie et la mort des images, les technologies de reproduction, les médias hybrides, ainsi que les médias et les humains en tant que collectifs donneurs de sens, également à l'ère numérique. Cela stimule la réflexion sur ce qui a été inscrit par qui, quand, et où dans les archives (numériques), et comment une mémoire visuelle particulière a été produite, défiée, contestée et transformée.

L'objectif de la série de livres est d'initier et d'encourager les débats et les échanges sur les images et les films en tant qu'objets matériels et sociaux comple-

Ә Open Access. (C) 2019 Karin Priem, published by De Gruyter. $($ BY-NC-ND This work is licensed under the Creative Commons Attribution-NonCommercial-NoDerivatives 4.0 License.

https://doi.org/10.1515/9783110655599-203 
xes. Cet objectif sera atteint en considérant les images comme des objets avec lesquels on pense, en les problématisant comme des signes ou des traces d'intrications complexes avec le passé et le présent.

Le premier volume de cette série est le résultat d'une collaboration fructueuse entre l'une des éditrices de la série, chercheuse aux Archives de l'UNESCO, et une historienne de la photographie, et témoigne du développement riche et enchevêtré de l'histoire des archives, de l'histoire de l'éducation, de la recherche visuelle et des politiques médiatiques des organisations humanitaires.

Esch-sur-Alzette, le 4 octobre 2019

Au nom des éditrices et éditeurs de la série,

Karin Priem 